\title{
TECNOLOGIAS PARA A CONTINUIDADE DO CUIDADO À DISTÂNCIA
}

\author{
Rosimere Ferreira Santana ${ }^{1}$ \\ Maria Auxiliadora Rodrigues ${ }^{2}$ \\ Ana Beatriz Serra Hercules ${ }^{3}$ \\ Carla Targino Bruno dos Santos ${ }^{4}$ \\ Tallita Mello Delphino5 \\ Thalita Gomes do Carmo ${ }^{6}$
}

resumo

Introdução: A tecnologia pode se apresentar como uma aliada dos profissionais da saúde para possibilitar a continuidade do cuidado em domicílio. A utilização dela se justifica pelo aumento da complexidade

1 Enfermeira. Doutora em Enfermagem. Professora Associada da Escola de Enfermagem Aurora de Afonso Costa, da Universidade Federal Fluminense (UFF), Niterói, RJ. E-mail: rfsantana@id.uff.br. 2 Enfermeira. Doutoranda em Ciências do Cuidado em Saúde. Escola de Enfermagem Aurora de Afonso Costa, da UFF, Niterói, RJ. E-mail: enfmado@gmail.com.

3 Enfermeira. Ex-bolsista PIBITI/Agir/Cnpq, Escola de Enfermagem Aurora de Afonso Costa da UFF, Niterói, RJ. E-mail: anabeatriz.absh@gmail.com.

4 Enfermeira. Ex-bolsista PIBITI/Agir/Cnpq, Escola de Enfermagem Aurora de Afonso Costa da UFF, Niterói, RJ. E-mail: anabeatriz.absh@gmail.com.

5 Enfermeira. Doutoranda em Ciências do Cuidado em Saúde. Escola de Enfermagem Aurora de Afonso Costa, da UFF, Niterói, RJ. E-mail: tallitamell@gmail.com.

6 Enfermeira. Doutora em Enfermagem. Professora Assistente da Escola de Enfermagem Aurora de Afonso Costa, da UFF, Niterói, RJ. E-mail: thalitado@gmail.com. 
do estado de saúde dos indivíduos que cada vez mais possuem doenças crônicas e agudas, a crescente expectativa de vida, a dificuldade de deslocamento nas grandes cidades e nas áreas rurais e, sobretudo, ao aumento da adesão as diversas redes sociais que possibilitam a facilidade e rapidez na comunicação. Objetivo: discutir estratégias do uso de tecnologias para a continuidade de cuidados à distância aos idosos. Métodos: foram apresentados três estudos de intervenção sobre a aplicabilidade de tecnologias para continuidade de cuidados à distância. Resultados: em pacientes submetidos a cirurgia de catarata, o telecuidado influenciou na diminuição do tempo de recuperação cirúrgica, na menor ocorrência de complicações e as intervenções mais realizadas foram referentes ao desconforto, proteção dos olhos, curativo e autocuidado. Nos pacientes submetidos a cirurgia cardíaca, observa-se que o discurso demonstra retorno ao hospital para acompanhamento dos exames de sangue, preocupação com a cicatriz cirúrgica, bom estado geral, apesar das dores, e satisfação por receber o contato via Whatsapp $®$. Sobre idosos com demencia e seus cuidadores, a ligação telefônica reduziu o risco para declínio funcional nos idosos e a sobrecarga dos cuidadores de idosos. Conclusão: o telecuidado foi testado e demonstrou resultados positivos em três cenários, mas destaca-se a necessidade de ampliar essa tecnologia em mais modalidades com outras tecnologias integradas que possam melhorar a qualidade de vida e autonomia dos idosos e familiares.

palavras-chave

Telecuidado. Tele-enfermagem. Telemedicina. Continuidade da Assistência ao Paciente. Enfermagem geriátrica.

Diante da mudança significativa nos quadros demográfico e epidemiológico no atual perfil de saúde da população idosa, com tripla carga de doenças, caracterizado pela concorrência de condições crônicas e agudas, somadas ainda aos agravos decorrentes de causas externas, se faz necessário a implantação de modelos de atenção que respondam adequadamente às condições crônicas e a longo prazo. A organização dos sistemas de saúde pode variar entre uma conjuntura de serviços fragmentados, até uma integração dos seus componentes. 
Em geral, sistemas fragmentados vinculam-se a modelos de atenção que respondem às condições agudas, enquanto sistemas integrados estão articulados aos modelos de atenção às condições crônicas (MENDES, 2018). A tecnologia, sob diversas modalidades, tem sido incorporada na gestão e assistência, com o intuito de atender às necessidades de cuidado emergentes à saúde da população (BARBOSA; SILVA, 2017). Para a enfermagem, o uso de tecnologias no cuidar baseia-se no conhecimento técnico e científico, na observação do cotidiano e na preocupação com o bem-estar, tanto do cuidador como do sujeito a ser cuidado (AQUINO et al., 2010; TEMOTEO et al., 2019).

As Tecnologias da Informação e Comunicação comumente aplicadas para continuidade do cuidado em domicílio tem sido o acompanhamento por ligações telefônicas, videoconferência e mensagens de celular via SMS (Short Message Service) ou via aplicativo de mensagens (ex: Whatsapp), plataformas digitais, sensores ou robôs com inteligência artificial, ou mesmo várias tecnologias associadas a presença física de programas ambulatoriais ou Homecare. O uso dessas tecnologias está relacionado ao fortalecimento de orientações recebidas durante a alta hospitalar, à velocidade de acesso do paciente ao profissional de saúde, à diminuição do tempo de espera para a consulta, à redução de tempo e custo na locomoção para consultas presenciais, além de possibilitar aumento na frequência dos contatos e facilitar o retorno do paciente (DELPHINO; SANTANA; HERCULES, 2020; DELPHINO; SOUZA; SANTANA, 2016; SANTANA et al., 2018). Além dessas, outras tecnologias também podem ser destacadas para a continuidade do cuidado, como o sistema de monitoramento noturno; sistema de monitorização e localização por GPS; assistência de navegação robotizada; e estímulos por comandos verbais (MAIA et al., 2018). O sistema de monitoramento noturno permite a redução do risco de ocorrência de quedas, possibilita maior tempo de descanso ao cuidador, diminuindo a sobrecarga, além de reduzir a probabilidade de institucionalização. O sistema de localização por GPS, por sua vez, aumenta a capacidade dos idosos de se deslocar de maneira independente e segura. Já os comandos verbais em forma de lembretes auxiliam a execução de atividades de controle financeiro e manuseio de medicamentos, e são ativados quando o idoso se comporta de forma a não saber dar continuidade à atividade. No tocante ao uso de auxiliares de navegação robóticos, os achados sustentam a afirmação de que estes são eficazes para apoiar a orientação e o deslocamento de pessoas com demência, além de serem capazes de reduzir o tempo de execução das tarefas (MAIA et al., 2018). Cada tecnologia adicionada ao processo de cuidar em enfermagem e aos sistemas organizacionais permite continuidade dos cuidados em domicílio, oferece ensino e orientações pertinentes conforme necessidade de 
cada paciente, melhora a qualidade do cuidado, diminui os eventos adversos e erros (TEMOTEO et al., 2019; DELPHINO; SOUZA; SANTANA, 2016). Para essas estratégias, alguns termos têm surgido na área da saúde: telessaúde, telemedicina, tele-enfermagem, telecuidado e telemonitoramento (DELPHINO; SOUZA; SANTANA, 2016; DELPHINO; SANTANA; HERCULES, 2020).

O termo telessaúde refere-se à prestação de serviços de atenção à saúde por meio do uso de tecnologias de informação e comunicação (TIC). Essa estratégia pretende superar a barreira da distância e aproximar os serviços de saúde; promover acesso e melhorar a qualidade da assistência com apoio à tomada de decisão pelos profissionais; compartilhar e coordenar recursos geograficamente distribuídos, otimizando seu uso e garantindo a oferta qualificada de diferentes especialidades em saúde (ORGANIZACIÓN PANAMERICANA DE LA SALUD, 2016). A telemedicina é uma categoria existente dentro da própria telessaúde, sendo a tecnologia que proporciona essa troca de informações entre médicos e pacientes, mesmo que à distância. Já o telecuidado caracteriza-se pelo uso das telecomunicações e das tecnologias computacionais na prestação de cuidados de enfermagem à distância, para o acompanhamento de adultos e idosos em domicílio, por meio de ligações telefônicas, vídeo conferência, consultas virtuais e/ou mensagens de celular. Tem se manifestado como uma tecnologia eficaz na modificação dos estilos de vida de pacientes em condições crônicas de saúde e cirúrgicas (DELPHINO; SANTANA; HERCULES, 2020; DELPHINO; SOUZA; SANTANA, 2016; SANTANA et al., 2018). Portanto, no telecuidado, ocorre uma centralidade do trabalho dos enfermeiros pela tradição no cuidado em atenção domiciliar, que reside na gestão da assistência ou na assistência direta (FURAKER, 2012). Destaca-se, ainda, que este profissional pode desempenhar papel fundamental, tanto pela coordenação do plano de cuidados no domicílio quanto pelo vínculo que estabelece com usuários e familiares. Ademais, esse protagonismo evidencia-se por proporcionar a articulação entre a família e a equipe multiprofissional, uma vez que, de modo geral, compete ao enfermeiro a capacitação do cuidador familiar, a supervisão dos técnicos de enfermagem e a identificação de demandas para outros profissionais (SILVA et al., 2015).

Dessa forma, o cuidado em saúde, em gerontologia e geriatria, em geral, se caracteriza por indivíduos com altas demandas de cuidado e diferentes níveis de dependência, causados por patologias e agravamentos que repercutem diretamente na capacidade funcional e autonomia (DIAS, 2017; CARNAÚBA et al., 2017). Assim, idosos e suas famílias vivenciam modificações importantes em seu cotidiano. Muitas vezes, necessitam realizar adaptações nos espaços físicos de seus lares e promover reorganizações financeiras e familiares, além de se 
contextualizarem acerca das alterações biopsicossociais geradas pela doença, passando pela aceitação da nova condição até a necessidade de aprendizado e adaptação frente ao uso de novos materiais e conhecimentos, bem como obtenção de disposição, habilidades e competências para o cuidado (SILVA; SENA; CASTRO, 2017; MOTA et al., 2018). Essas mudanças acomodam-se ao conceito de Transição, descrito pelas enfermeiras Chick e Meleis (1986), que elaboraram um conceito de transição aplicável para a ciência da enfermagem. Para elas, transição consiste em "uma passagem de um estado, condição ou local, para outro" (CHICK; MELEIS, 1986, p. 239). Está associada ao processo de viver, é inevitável, mas nem sempre é natural ou desejada, logo, exige dos indivíduos inseridos a incorporação de conhecimentos, alteração de práticas e comportamentos e até a redesignação de si (self). O processo de transição é concluído quando a pessoa readquire estabilidade, visualizada por indicadores, como bem-estar subjetivo, nas relações e no desenvolvimento satisfatório de seus papéis sociais (FAVERO, 2013).

Os enfermeiros que promovem o cuidado transicional valorizam o indivíduo, já que os cuidados dispensados estão associados ao desenvolvimento humano, beneficiando a maturidade e o crescimento pessoal mediante um maior equilíbrio e estabilidade (MELEIS, 2007). Sendo assim, é importante ressaltar que uma assistência em telecuidado personalizada, sustentada na teoria das transições que considerem os processos adequados que possam otimizar indicadores assistenciais e contribuir para a diminuição das taxas de complicações e reinternações são fundamentais para o sucesso de uma aplicação tecnológica. Além do fator humano, os profissionais de saúde capacitados para compressão da ferramenta tecnológica (melhor TIC para cada caso), do processo de cuidado (teoria das transições) e da pessoa (gerontologia), isso, em conjunto, é fundamental para o desenho de uma aplicação tecnológica para a continuidade do cuidado em domicílio. Dessa forma, este estudo teve por objetivo: discutir estratégias do uso de tecnologias para a continuidade de cuidados à distância aos idosos.

\section{Método}

Serão apresentados a seguir exemplos da aplicabilidade de tecnologias para continuidade de cuidados à distância, nas seguintes situações: 1. Acompanhamento telefônico de idosos em pós-operatório de cirurgia de catarata; 2. Acompanhamento via WhatsApp ${ }^{\circledR}$ de pacientes em pós operatório de cirurgia cardíaca; e 3 . Acompanhamento por telefone e vídeo conferência de idosos com Alzheimer e seus cuidadores. 


\subsection{Acompanhamento telefônico de idosos em pós-operatório de cirurgia de catarata}

\section{Método utilizado:}

Realizou-se um ensaio clínico randomizado com 95 pacientes de ambos os sexos, maiores de 60 anos e submetidos a cirurgia de catarata, provenientes de dois hospitais localizados no município de Niterói - RJ, no período de agosto de 2016 a fevereiro de 2017. Os pacientes foram divididos em um grupo de intervenção (48 pacientes) e um grupo controle (47 pacientes). Foram estabelecidos os seguintes critérios: 1) Critérios de Inclusão: possuir 60 anos ou mais de idade, estar em pós-operatório de cirurgia de catarata e possuir telefone celular ou fixo disponível para o contato pela enfermeira. 2) Critérios de Exclusão: presença de demência ou com déficit auditivo sem acompanhante ou familiar que pudesse receber as intervenções telefônicas, e pacientes submetidos a cirurgias para tratamento de complicações cirúrgicas prévias. 3) Critérios de Descontinuidade: atender menos de $75 \%$ das chamadas telefônicas e não disponibilizar tempo para as orientações ao telefone.

As consultas presenciais no ambulatório de oftalmologia foram mantidas e ocorreram no primeiro, sétimo e trigésimo dia de pós-operatório, com o fornecimento de orientações sobre os cuidados no pós-operatório e avaliação do olho operado. $\mathrm{O}$ acompanhamento por telefone consistiu em quatro ligações, realizadas no $1^{\mathrm{o}}$ dia de pós-operatório (primeira ligação efetuada para o contato inicial), $4^{\mathrm{o}}$ dia de pós-operatório (segunda ligação), 10ํำ dia de pós-operatório (terceira ligação) e $20^{\circ}$ dia de pós-operatório (quarta ligação). As ligações foram realizadas na central de telecuidado, no horário comercial durante a semana, e das 8 às $12 \mathrm{~h}$ aos sábados. Durante as ligações, foi utilizado um software on-line para realização da entrevista e preenchimento do protocolo de ligação, que consistiu em um formulário semiestruturado contendo perguntas sobre a recuperação pós-operatória e orientações referentes aos cuidados em domicílio.

\section{Principais resultados:}

O grupo acompanhado pelo telecuidado apresentou diminuição no tempo de recuperação cirúrgica $(\mathrm{p}=0,000)$. A ocorrência de complicação cirúrgica no grupo intervenção foi de $6,3 \%$, enquanto, no grupo controle, foi de $36,2 \%$, com razão de chance $(\mathrm{OR})=0,118$; intervalo de confiança $(\mathrm{IC})=0,032 ; 0,437$. 
Em relação às principais intervenções realizadas durante o acompanhamento por telefone, observou-se uma demanda maior de orientações nos primeiros dias de pós-operatório, principalmente referente ao controle do desconforto, uso do tampão ocular, limpeza do olho operado, utilização do colírio, uso dos óculos escuros e orientações quanto ao autocuidado. À medida que os cuidados foram sendo compreendidos e incorporados aos hábitos diários, a demanda de orientações também diminuiu.

O acompanhamento por telefone demonstrou-se como significativa intervenção de enfermagem no pós-operatório de idosos submetidos a cirurgia de catarata, pois permitiu orientações ao paciente em domicílio, diagnóstico precoce das necessidades de saúde, prevenção de doenças e complicações, maior adesão aos tratamentos propostos e aos cuidados em domicílio (DELPHINO; SANTANA; HERCULES, 2020; DELPHINO; SOUZA; SANTANA, 2016; SANTANA et al., 2018). Os contatos telefônicos permitiram o acompanhamento do paciente após a alta hospitalar, bem como a realização de orientações importantes para a recuperação cirúrgica, independência e autonomia do idoso.

\subsection{Acompanhamento via WhatsApp de pacientes em pós-operatório de cirurgia cardíaca}

Método utilizado:

Realizou-se um estudo descritivo para analisar a conversa via WhatsApp ${ }^{\circledR}$ de 81 pacientes adultos e idosos submetidos a cirurgia cardíaca no Instituto Nacional de Cardiologia, Rio de Janeiro, RJ, no período de julho de 2017 a agosto de 2018, que, originalmente, contou com 181 pacientes e teve como critério de elegibilidade pacientes que receberam alta hospitalar e que responderam a pelo menos um dos três contatos realizados nos 30 dias após a alta via WhatsApp ${ }^{\circledR}$ (CARMO, 2018). A coleta de dados ocorreu em três fases: $1^{\mathrm{a}}$ inclusão do paciente, com a visita pré-operatória com assinatura do termo de consentimento livre e esclarecido e coleta de dados com os instrumentos dessa fase; $2^{2}$ visita pós operatória com nova coleta de dados com os instrumentos dessa fase; $3^{\text {a }}$ contato

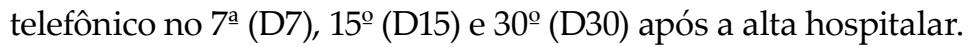

Dos 181 pacientes, 13 foram a óbito intra-hospitalar, um foi transferido para outra instituição e 167 receberam alta hospitalar. Desses 167, realizou-se contato telefônico, sendo que 86 responderam o contatado via telefone e 81 pacientes via WhatsApp ${ }^{\circledR}$, resultando em 119 conversas dos três contatos. As perguntas realizadas via WhatsApp ${ }^{\circledR}$ foram: “Como têm sido esses 7 ou 15 ou 30 dias pós 
alta hospitalar?"; "Retornou ao hospital para readmissão por algum motivo?"; “Tem caminhado?"; “Tem se alimentado?"; "A cicatriz cirúrgica está fechada?". Essas perguntas foram feitas e sustentadas pelo diagnóstico de enfermagem Recuperação cirúrgica retardada (HERDMAN; KAMITSURU, 2018). Para analisar as conversas, foi criado um programa de computador baseado em um algoritmo que contabiliza a frequência das palavras que mais se repetem nos textos gerando uma nuvem de palavras. Foram excluídas da contagem o protocolo de mensagem enviado a todos os pacientes, pois este aparece em todas as conversas mais de uma vez. As palavras das conversas foram divididas em unigram, uma única palavra; bigram, duas palavras; e trigram, três palavras.

\section{Principais resultados:}

Os participantes do estudo eram maioria do sexo masculino (56,8\%), com média de idade de 58,1 anos, com desvio padrão de 9,9. A principal cirurgia realizada foi a de revascularização do miocárdio com circulação extracorpórea (45,7\%), seguida por troca valvar múltipla ou combinada com plastia $(17,3 \%)$ e troca/plastia de aorta (14,8\%). O Número de dias totais de internação foi em média de 35,7 dias, com desvio padrão de 18,1. As nuvens de palavras foram organizadas de três formas: a primeira nuvem (Figura 1) com palavras únicas e desassociadas de outras palavras, chamadas de unigram; a segunda (Figura 2) com duas palavras seguidas e associadas, chamadas de bigram; e a terceira (Figura 3) com três palavras seguidas e associadas, chamadas de trigram. As palavras que se sobressaem em frequência são as que estão maiores em tamanho. 
Figura 1 - Nuvem unigram

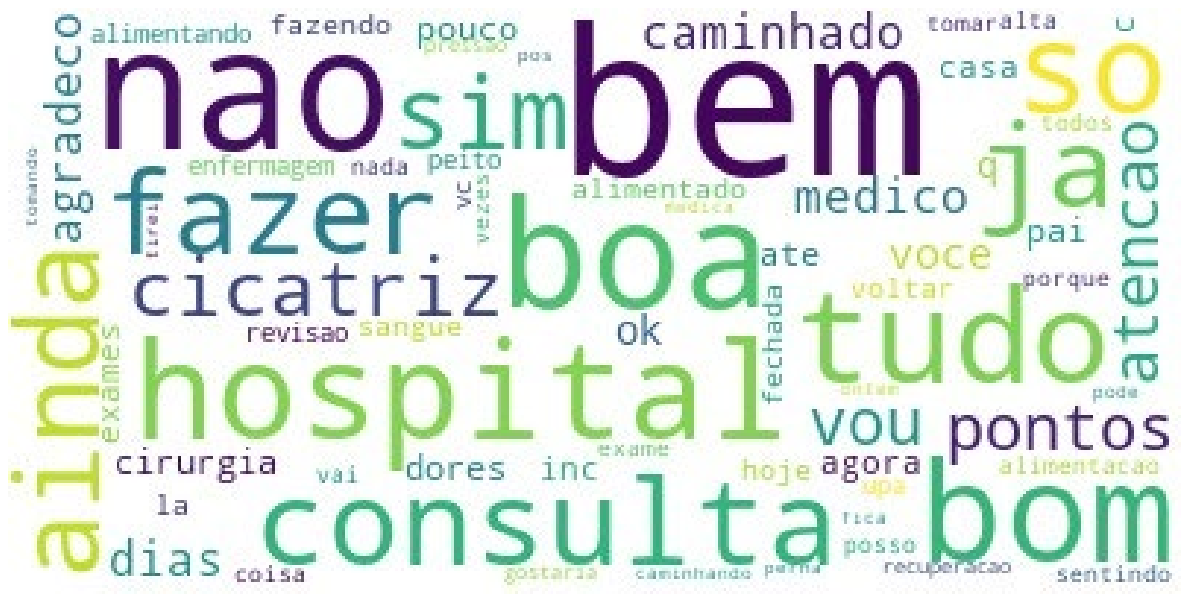

Fonte: Elaborada no programa criado pelos autores.

A nuvem unigram apresenta palavras soltas que são de difícil conexão, porém percebe-se que os pacientes relatam sobre o hospital, consultas e a cicatriz.

Figura 2 - Nuvem bigram

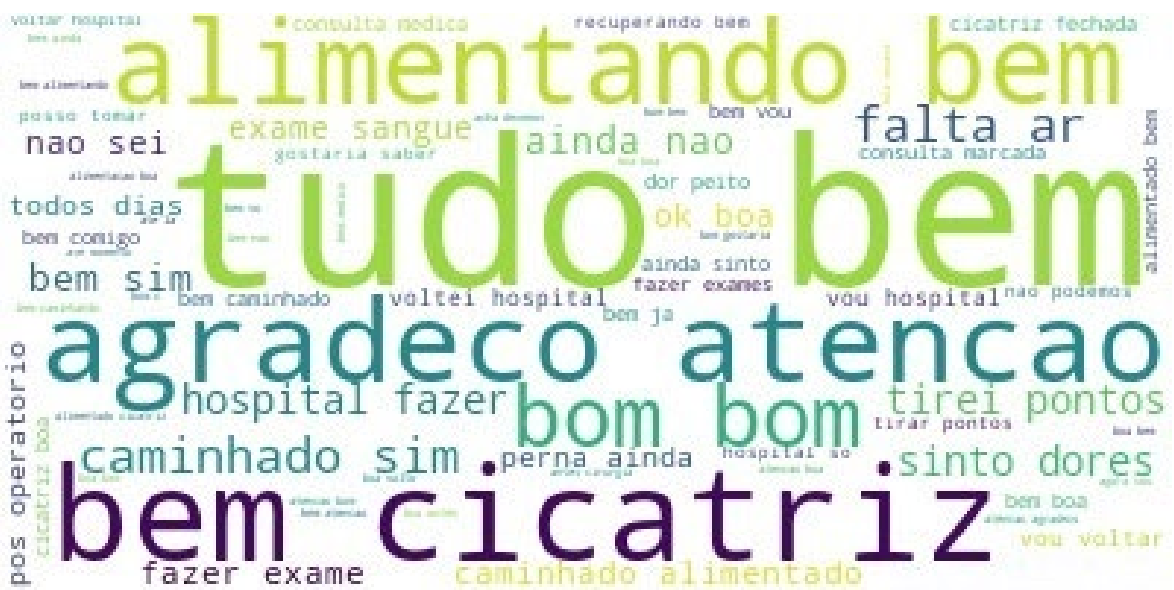

Fonte: Elaborada no programa criado pelos autores.

Os resultados da nuvem bigram demonstram que os pacientes têm se alimentado bem, que a cicatriz cirúrgica está com boa evolução e já retiram 
os pontos e que o estado geral deles é estável. Há destaque para o retorno ao hospital, o que é protocolo do Instituto Nacional do Coração para acompanhamento, e que alguns deles ainda sentem dores.

Figura 3 - Nuvem trigram

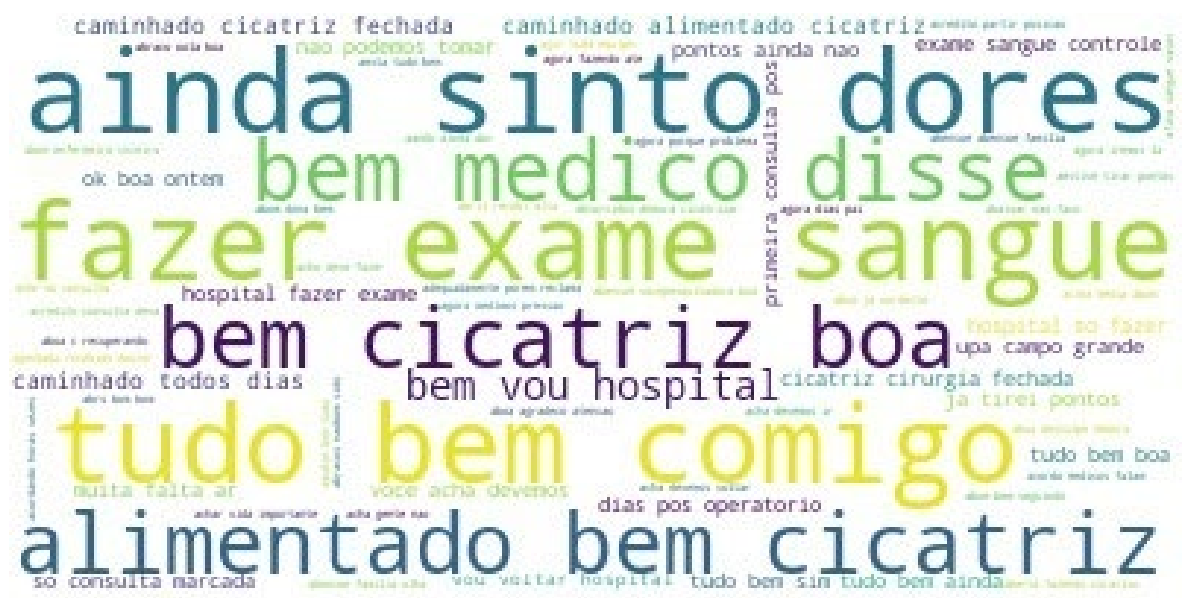

Fonte: Elaborada no programa criado pelos autores.

Os resultados da nuvem trigram estão em consonância com a bigram, com relato de dores, bom estado geral e acompanhamento hospitalar com realização de exames de sangue de rotina. O destaque dessa nuvem é o relato de falta de ar e da boa recuperação. As nuvens de conversas geradas demonstraram que os pacientes tiveram boa aceitação e gratidão ao receber o contato via mensagens instantâneas, estão com uma boa recuperação cirúrgica, continuam fazendo acompanhamento no Instituto Nacional de Cardiologia e outros possuem queixas que carecem de intervenção de enfermagem e saúde. As complicações pós operatórias tardias podem acontecer até mesmo em 30 dias após a cirurgia, alterando o quadro clínico do paciente. Atualmente, os pacientes submetidos ao procedimento cirúrgico deveriam ter alta cada vez mais precoce. As dúvidas no âmbito hospitalar são constantes e o retorno para casa, em que o paciente ou familiar será responsável pelo cuidado, causa medo e ansiedade. Essa situação propicia a criação de um planejamento de um programa de acompanhamento para os pacientes. Ouso do telefone de modo tradicional para seguimento no pós-alta hospitalar foi estudado por autores em outras cirurgias e demonstraram bons resultados (BECCARIA et al., 2015; CARVALHO; MAMEDE; ARAÚJO, 2011; CARDOZO et al., 2017; LIMA et al., 2018; DELPHINO; SOUZA; SANTANA, 2016). 
Os perfis dos participantes se tornam complexos a cada dia com cirurgias extensas e em uma população cada dia mais envelhecida, com dificuldade de deslocamento pelas distâncias e complexidade urbana, ou mesmo pela necessidade de acompanhamento de um familiar. Com isso o uso de tecnologias no cotidiano, viabilizam o acompanhamento de pacientes via WhatsApp ${ }^{\circledR}$ de pacientes de cirurgia cardíaca. Os pacientes se mostraram familiarizados com uso do aplicativo e satisfeito com o atendimento.

\subsection{Acompanhamento por telefone e vídeo chamada a idosos com Alzheimer e seus cuidadores}

\section{Método utilizado:}

Tratou-se um estudo experimental, de abordagem quantitativa para avaliação do efeito do telecuidado pela enfermeira no acompanhamento de idosos com demência e seus cuidadores. O cenário de pesquisa foi o Centro Multidisciplinar do Idoso (CMI) do Hospital Universitário de Brasília (HUB), que consiste em um centro referência para o atendimento de demências no Distrito Federal. São disponibilizados para a população serviços ambulatoriais nas especialidades de Geriatria Geral e Demência aos idosos do DF, por meio de consulta, acompanhamento ambulatorial e visitas domiciliares. Foram selecionados como participantes idosos frequentadores do CMI e seus cuidadores. Como critérios de inclusão foram selecionados idosos com idade igual ou superior a 65 anos, possuir cuidador principal e estar em acompanhamento pelo CMI/HUB e com pontuação do Mini Exame do Estado Mental (MEEM) menor ou igual que 26 . Os cuidadores precisavam possuir disponibilidade para orientações e capacidade cognitiva de resposta orientada no tempo e espaço para esta interface com a pesquisadora.

Foram exclusos idosos que possuíam instabilidades clínicas como balão de oxigênio, insuficiência cardíaca congestiva descompensada, doença pulmonar obstrutiva congestiva, doenças cardiovasculares, doenças cerebrovasculares, doenças psiquiátricas e estar em cuidados paliativos avançados e cuidadores com déficit auditivo que limitassem a comunicação por telefone e \ou vídeochamadas. A amostra total foi composta por 40 participantes. Após aceite em participar da pesquisa e assinatura do Termo de Consentimento Livre e Esclarecido, os idosos e cuidadores foram randomizados de forma aleatória e simples, por uma sequência elaborada pelo Software Bioestat versão 5.3, que gerou uma sequência que depois de incluídos na amostra que comporão o grupo controle e os que serão destinados ao grupo experimento, sem influência do pesquisador. 
Houveram 24 (60\%) participantes idosos do sexo feminino, com média de idade de 82,5 anos, 18 (45\%) com Doença de Alzheimer, grau de demência segundo CDR - moderada 23 (57,5\%) e Prisma-7 36 (90\%) com risco de fragilidade. Os cuidadores que receberam as ligações foram maioria do sexo feminino $29(72,5 \%)$, sendo que os filhos foram os que mais atenderam as ligações 26 (65\%), seguido de conjugue $11(27,5 \%)$, e sobrinhas três $(7,5 \%)$. O telecuidado foi realizado por um tempo de seguimento de três meses, através de um instrumento de ligação construído visando apoiar o cuidador no manejo das atividades de cuidado junto ao idoso e reduzir sua sobrecarga. As ligações foram realizadas quinzenalmente por um período de 90 dias e no final do tempo de seguimento, os participantes foram novamente avaliados no CMI/HUB, com os mesmos instrumentos da avaliação inicial (PRISMA, CDR, ZARIT, CAM e WHOQOL BREEF).

Em relação a duração das ligações, obteve-se que nas quatro primeiras ligações foram as mais longas, variaram de 40 a 70 minutos, com duração mediana de 51 minutos. A partir da quinta ligação a duração da ligação tendeu a diminuir, a ponto de na décima quarta e última ligação, variarem de 20 a 40 minutos, com duração mediana de 25 minutos, média de 27,5 minutos e desvio padrão de 5,5 minutos, que representa moderada variabilidade da duração destas ligações $(\mathrm{CV}=0,20)$.

Os resultados demostraram em relação à medida PRISMA um efeito significativo na intervenção, houve diferença entre os grupos no período final ( $\mathrm{p}$-valor=0,01), a presença de risco no grupo intervenção (45\%) foi menor do que o grupo controle (88,2\%). Portanto, o acompanhamento telefônico realizado pela Enfermeira, intervindo junto ao cuidador familiar, reduziu o risco para declínio funcional nos idosos, uma vez abordou orientações voltadas para as principais necessidades da pessoa com demência e para seus cuidadores familiares quanto ao cuidado e recursos de controle do estresse e sobrecarga, conferindo ao cuidador familiar instrumentos para o manejo das alterações biológicas, psicológicas e comportamentais no cotidiano de cuidado. Estudos demonstram que a intervenção da enfermeira por meio de acompanhamento por telefone melhora a adesão ao tratamento, as condições clínicas, autonomia e independência do idoso em pós-operatório de catarata (SANTANA et al., 2018; CARDOZO et al., 2017).

Os dados do CAM (Confusion Assessment Method) foram maiores no grupo controle, que o grupo intervenção apoiado pela Enfermeira, mas esses não tiveram uma diferença estatística significativa. Os sintomas como alucinação, agitação e ansiedade no idoso reduziram com a intervenção, ou seja, o cuidador familiar esteve mais preparado para lidar com estes sintomas, reduzindo a sua ocorrência, 
esse resultado indiretamente melhora a sobrecarga e qualidade de vida do cuidador. A intervenção por meio de acompanhamento por telefone realizada pela enfermeira, nas condições propostas por este estudo, reduziu a sobrecarga dos cuidadores familiares. Um estudo sobre as intervenções de enfermagem para apoiar cuidadores familiares em cuidados de final de vida em casa mostrou-se positivo, principalmente as relacionadas às intervenções multicomponentes que foram mais bem-sucedidas: psicoeducação, avaliação de necessidades, suporte prático com o cuidado e apoio de amigos (BECQUÉ et al., 2019).

A qualidade de vida dos cuidadores familiares de idosos que não receberam a intervenção revela piora nas relações sociais. Isso porque o acompanhamento telefônico realizado pela Enfermeira auxiliou no contexto social dos cuidadores familiares de idosos com demência, apoiando e incentivando as relações sociais, e sendo a própria interação, ou seja, reduz a sensação de isolamento do cuidador referente ao cuidado. A demência leva a uma dependência progressiva do idoso, levando ao cuidador familiar a abdicar de suas relações sociais, atividades e outras relações familiares ocasionando sobrecarga física, emocional, financeira, dentre outros domínios.

Foi investigado ainda se o melhoramento observado nas escalas estava significativamente associado ao tipo de ligação, se houve diferença no melhoramento no grupo que recebeu ligação por vídeo-chamada através do WhatsApp ${ }^{\circledR}$ e no grupo que recebeu ligação por voz. Entretanto, não houve diferença significativa entre as duas proporções ( $\mathrm{p}$-valor $=1,000$ do teste Exato de Fisher). $\mathrm{O}$ mesmo se conclui para o melhoramento do Zarit e do PRISMA, que não estão significativamente associados ao tipo de ligação que o paciente recebeu, ou seja, o melhoramento observado independe do tipo de ligação.

\section{Considerações finais}

Foram apresentados três exemplos de aplicação do telecuidado para tipos diferentes de pacientes idosos que requeriam continuidade do cuidado, tanto no pós-operatório de catarata - telefone - como para cirúrgica cardíaca WhatsApp ${ }^{\circledR}$ - e para idosos com demência - telefone e videochamadas. Esses foram sustentados no cuidado transicional, compreendendo a importância de um protocolo de ligação personificado, com profissionais enfermeiros especializados e na importância de avaliar a melhor tecnologia para cada caso e aplicação. Por isso, recomenda-se investimento em gerontecnologia no cuidado aos idosos, com necessidade de novos testes com modalidades diferentes e com uso de tecnologias integradas que melhore a performance dessas aplicações para melhora da qualidade de vida e autonomia dos idosos e família. 
abstract

Introduction: Technology may present itself as an ally to health professionals to enable continuity care at home. Its use is justified by the increase of health condition complexity, which includes increased prevalence of chronic and acute diseases, growing life expectancy, difficulty of commuting in large cities and rural areas and, mainly, increased adherence to the various social media that make communication easier and faster. Purpose: discuss studies that use technology for continuity of distance care Methods: there are three Interventions studies about the applicability of technology for continuity of long-distance care. Results: In patients undergoing cataract surgery, the long-distance care made a difference in decreasing time in surgery recovery, less appearance of complications and the most commons interventions were about discomfort, eyes protection, bandage and selfcare. In patients undergoing cardiac surgery, it was observed that the speech was about returning to the hospital for blood tests, worry about the surgery scar, good health conditions, concerns for the pain, and satisfaction for receiving messages from Whatsapp $®$. About the elderly people with dementia, the phone call reduced the risk of functional decline in the elderly and caregiver burden. Conclusion: The long-distance care was tested and showed positives results in three different scenarios but highlights the need to expand this technology in more ways with other integrated technologies that can improve quality of life and independence of the elderly and their families.

keywords

Long-distance care. Long-distance nursing. Long-distance medicine. Continuity of Patient Care. Geriatric nursing.

Referências

AQUINO, Priscila de Souza; MELO, Renata Pereira de; LOPES, Marcos Venícius de Oliveira; PINHEIRO, Ana Karina Bezerra. Análise do conceito de tecnologia na enfermagem segundo o método evolucionário. Acta Paulista de Enfermagem, São Paulo, v. 23, n. 5, p. $690-696,2010$.

BARBOSA, Ingrid Almeida; SILVA, Maria Júlia Paes da. Cuidado de enfermagem por telessaúde: qual a influência da distância na comunicação? Revista Brasileira de Enfermagem, Brasília, DF, v. 70, n. 5, p. 978-984, set./out. 2017. 
BECCARIA, Lucia Marinilza; CESARINO, Claudia Bernardi; WERNECK, Alexandre Lins; GÓES, Nadielly Codonho; SANTOS, Karla Soares dos; MACHADO, Maurício de Nassau. Complicações pós-operatórias em pacientes submetidos à cirurgia cardíaca em hospital de ensino. Arquivos de Ciências da Saúde, São José do Rio Preto, v. 22, n. 3, p. 37-41, jul-set. 2015 .

BECQUÉ, Yvonne; RIETJENS, Judith; DRIEL, Anne Geert van; HEIDE, Agnes van der; WITKAMP, Erica. Nursing interventions to support family caregivers in end-of-life care at home: a systematic narrative review. International Journal of Nursing Studies, United Kingdom, v. 97, p. 28-39, set. 2019

CARDOZO, Aluane dos Santos; SANTANA, Rosimere Ferreira; ROCHA, Isamara da Conceição Moraes da; CASSIANO, Keila Mara; DELPHINO, Tallita Mello; MELO, Uyara Garcia. Acompanhamento por telefone como intervenção de enfermagem na recuperação cirúrgica de idosos prostatectomizados. Revista de Enfermagem UFPE Online, Recife, v. 11, n. 8, p. 3005-3012, 2017.

CARMO, Thalita Gomes do. Validação clínica dos diagnósticos de enfermagem risco de recuperação cirúrgica retardada e recuperação cirúrgica retardada em pacientes de cirurgia cardíaca. 2018. 180 f. Tese (Doutorado em Ciências do Cuidado em Saúde) - Escola de Enfermagem Aurora de Afonso Costa, Universidade Federal Fluminense, Niterói, 2018.

CARNAÚBA, Carla Montenegro Dâmaso; SILVA, Thaysa Dayse Alves e; VIANA, Juliana Felizardo; ALVES, Júlia Badra Nogueira; ANDRADE, Natália Lima; TRINDADE FILHO, Euclides Maurício. Caracterização clínica e epidemiológica dos pacientes em atendimento domiciliar na cidade de Maceió, AL, Brasil. Revista Brasileira de Geriatria e Gerontologia, Rio de Janeiro, v. 20, n. 3, p. 353-363, 2017.

CARVALHO, Líscia Divana; MAMEDE, Marli Villela; ARAUJO, Maria dos Remédios Oliveira de. Conhecimento de pacientes sobre o processo de auto-cuidado em pós-operatório de cirurgia cardíaca. Cadernos de Pesquisa, São Luís, v. 18, n. especial, p. 18-25, dez. 2011.

CHICK, Norm; MELEIS, Afaf Ibrahim. Transitions: a nursing concern. In: CHINN, Peggy. Nursing research methodology. Rockville: Aspen, 1986. p. 237-257.

DELPHINO, Tallita Mello; SANTANA, Rosimere Ferreira; HERCULES, Ana Beatriz Serra. Central de telecuidado: perspectiva de intervenção de enfermagem. Revista Cogitare Enfermagem, Curitiba, v. 25, p. e66666, 2020.

DELPHINO, Tallita Mello; SOUZA, Priscilla Alfradique de; SANTANA, Rosimere Ferreira. Telemonitoramento como intervenção no pós-operatório de facectomia: revisão sistemática da literatura. Revista Mineira de Enfermagem, Belo Horizonte, v. 20, p. e937, 2016.

DIAS, Jane Fonseca; GROSSI, Julia Baldoni; COSTA, Luciana Assis; FURTADO, Sheyla Rossana Cavalcanti; MANCINI, Marisa Cotta; SAMPAIO, Rosana Ferreira. Atenção domiciliar no âmbito da reabilitação e prática centrada na família: aproximando teorias para potencializar resultados. Revista de Terapia Ocupacional da Universidade de São Paulo, São Paulo, v. 28, n. 2, p. 206-213, 2017.

FAVERO, Luciane. Construção de um modelo de cuidado transpessoal em enfermagem domiciliar a partir do processo de cuidar. 2013. 173 f. Tese (Doutorado em Enfermagem) - Programa de Pós-Graduação em Enfermagem, Setor de Ciências da Saúde, Universidade Federal do Paraná, Paraná, 2013.

FURAKER, Carina. Registered nurses' views on competencies in home care. Home Health Care Management \& Practice, United States, v. 24, n. 5, p. 221-227, 2012.

HERDMAN, Tracy Heather; KAMITSURU, Shigemi. Diagnósticos de enfermagem da NANDA-I Internacional: definições e classificação (2018-2020). 11. ed. Porto Alegre: Artmed, 2018.

LIMA, Ivana Cristina Vieira de; GALVÃO, Marli Teresinha Gimeniz; PEDROSA, Samila Citó; CUNHA, Gilmara Holanda da; COSTA, Ana Karoline Bastos. Uso de aplicación Whatsapp para seguimento de salud de personas com VIH: un análisis temático. Escola Anna Nery Revista de Enfermagem, Rio de Janeiro, v. 22, n. 3, p. e20170429, abr. 2018. 
MAIA, Juliana Cunha; COUTINHO, Janaína Fonseca Victor; SOUSA, Caroline Ribeiro de; BARBOSA, Rachel Gabriel Bastos; MOTA, Fernanda Rochelly do Nascimento; MARQUES, Marilia Braga; SILVA, Rávida da Rocha Lima; LIMA, Rayanne Branco dos Santos. Tecnologias assistivas para idosos com demência: revisão sistemática. Acta Paulista de Enfermagem, São Paulo, v. 31, n. 6, p. 651-658, 2018.

MELEIS, Afaf Ibrahim. Theorical nursing: development e progress. 4. ed. Philadelphia: Lippincott, 2007.

MENDES, Eugênio Vilaça. Entrevista: a abordagem das condições crônicas pelo Sistema Único de Saúde. Ciência \& Saúde Coletiva, Rio de Janeiro, v. 23, n. 2, p. 431-436, 2018.

MOTA, Daniele de Norões; TORRES, Raimundo Augusto Martins; GUIMARÃES, José Maria Ximenes; MARINHO, Mirna Neyara Alexandre de Sá Barreto; ARAÚJO, Aretha Feitosa de. Tecnologias da informação e comunicação: influências no trabalho da estratégia Saúde da Familia. Journal of Health Informatics, São Paulo, v. 10, n. 2, p. 45-49, 2018.

ORGANIZACIÓN PANAMERICANA DE LA SALUD. Marco de implementación de un servicio de telemedicina. Washington, DC: OPS, 2016.

SANTANA, Rosimere Ferreira; DANTAS, Raquel Vaqueiro; SOARES, Thais da Silva; DELPHINO, Tallita Mello; HERCULES, Ana Beatriz Serra; LEITE JUNIOR, Homero Marinho Teixeira. Telecuidado para idosos com Alzheimer e seus cuidadores: revisão sistemática. Ciência, Cuidado e Saúde, Maringá, v. 17, n. 4, p. e41653, out./dez. 2018

SILVA, Juliana Paiva Góes da; COSTA, Kátia Nêyla de Freitas Macêdo; SILVA, Grazielle Roberta Freitas da; OLIVEIRA, Simone Helena dos Santos; ALMEIDA, Paulo César de: FERNANDES, Maria das Graças Melo. Consulta de enfermagem a idosos: instrumentos da comunicação e papéis da enfermagem segundo Peplau. Escola Anna Nery Revista de Enfermagem, Rio de Janeiro, v. 19, n. 1, p. 154-161, 2015.

SILVA, Kênia Lara; SENA, Roseni Rosângela de; CASTRO, Wesley Souza. A desospitalização em um hospital público geral de Minas Gerais: desafios e possibilidades. Revista Gaúcha de Enfermagem, Porto Alegre, v. 38, n. 4, p. e67762, 2017.

TEMOTEO, Rayrla Cristina de Abreu; CARVALHO, Jovanka Bittencourt Leite de; LIRA, Ana Luisa Brandão de Carvalho; LIMA, Maria Alzete de; SOUSA, Yanna Gomes de. Enfermagem na adesão ao tratamento da tuberculose e tecnologias em saúde no contexto da atenção primária. Escola Anna Nery Revista de Enfermagem, Rio de Janeiro, v. 23, n. 3, p. e20180321, ago. 2019.

Recebido: 25/04/2020

Aceito:30/05/2020 